\title{
Prenatal Diagnosis of Proximal Femoral Focal Deficiency Combining Ultrasound and Computer Tomography
}

\author{
Erica Stein Ciasca ${ }^{1}$, Fernando Maia Peixoto-Filho ${ }^{2}$, Pedro Daltro ${ }^{3}$, \\ Heron Werner ${ }^{4}$, Adriana Viana ${ }^{4}$, Renato Augusto Moreira de Sá ${ }^{1}$ \\ ${ }^{1}$ Universidade Federal Fluminense-NEASMI/PGCM, Niterói, Brazil \\ ${ }^{2}$ Department of Obstetrics-Instituto Fernandes Figueira-IFF/FIOCRUZ, Rio de Janeiro, Brazil \\ ${ }^{3}$ Instituto Fernandes Figueira-IFF/FIOCRUZ, Rio de Janeiro, Brazil \\ ${ }^{4}$ Clínica de Diagnóstico Por Imagem-CDPI, Rio de Janeiro, Brazil \\ Email: cacastein@gmail.com
}

Received November 15, 2012; revised December 16, 2012; accepted January 5, 2013

Copyright (c) 2013 Erica Stein Ciasca et al. This is an open access article distributed under the Creative Commons Attribution License, which permits unrestricted use, distribution, and reproduction in any medium, provided the original work is properly cited.

\begin{abstract}
Four cases of proximal femoral focal deficiency (PFFD) in an otherwise healthy infant are described. Antenatal diagnosis was made at 27, 23, 23 and 18 weeks of gestation by routine ultrasound (US) examination. Computer Tomography (CT) was performed after 30 weeks of gestation and confirmed the images obtained by US. The diagnosis was confirmed after delivery. These cases illustrate the importance of combining US and CT to improve accuracy of prenatal diagnosis of skeletal disorders.
\end{abstract}

Keywords: Prenatal Diagnosis; Ultrasonography; Imaging, Three-Dimensional; Tomography, X-Ray Computed; Limb Deformities; Congenital

\section{Introduction}

Estimating congenital short femur, or proximal femoral focal deficiency (PFFD), is a congenital anomaly of the pelvis and proximal femur. The deficiency results from failure in the development of the subtrochanteric portion of femoral shaft [1]. It is a rare condition with the incidence ranging between 0.11 and $0.2 / 10,000$ live births [2,3]. Prenatal screening and diagnosis of skeletal dysplasias are based on sonographic and radiological findings.

We report four cases of PFFD suspected by 2D-US and subsequently submitted to Computed Tomography (CT). Diagnostic confirmation was obtained by postnatal $\mathrm{X}$-ray and physical examination after birth. In this case report, we aim to discuss the contribution of new methods for the diagnosis of PFFD.

\section{Case Report}

Four cases of proximal femoral focal deficiency (PFFD) (18-, 33-, 32-, and 35-years old pregnant women; presentation at 27, 23, 23 and 18 weeks of gestation; 1 to 0,3 to 2,2 to 1 , and 1 to 0 ; female, male, female, and male fetuses, respectively), identified by routine ultrasound (US) examination in otherwise healthy infants are described. The family history was negative for congenital anomalies and no consanguinity was reported. The patients were not shown to have insulin-dependent diabetes mellitus. Prenatal exams performed in the first trimester of pregnancy showed normal results. The right femur was shorter than the left one in the second and fourth cases and the left femur was shorter than the right one in the first and third cases (Figure 1). Further sonographic exploration demonstrated unilateral femoral hypoplasia without another anomaly. In these cases, occurrence of PFFD was sporadic. Computer Tomography (CT) was performed after 30 weeks of gestation using a multislice 64 scanner (Philips, Solingen, Germany) with the following parameters: $40 \mathrm{~mA}, 120 \mathrm{kV}, 64$ slices per rotation, pitch 0.75 and slice thickness $0.75 \mathrm{~mm}$. This corresponds to a mean radiation dose to the fetus of $3.12 \mathrm{mGy}$ (CT dose index weighted) [4,5]. The acquisition lasted around $20 \mathrm{~s}$ and was performed during maternal apnea (Figure 2). The CT confirmed the images obtained by 2D-US and 3D-US (Figure 3). The presumptive diagnosis was congenital isolated short femur.

Elective cesarean sections were carried out at 38 weeks' for the first, second and fourth fetuses and 39 weeks' for the third fetus. The Apgar scores at 1 and 5 minutes were 9 and 10, respectively, for first, second and third newborn, and 7 and 9 for the fourth fetus. The weight at 


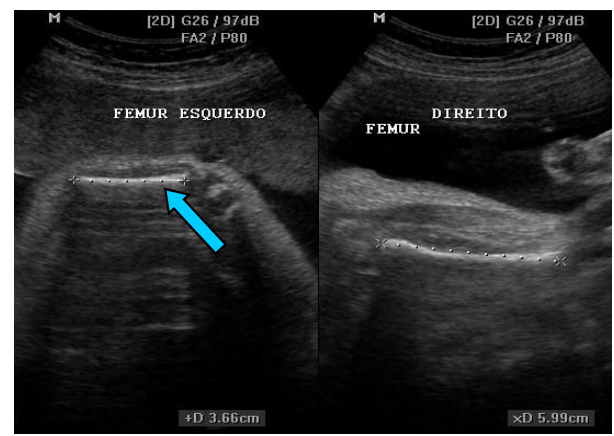

(a)

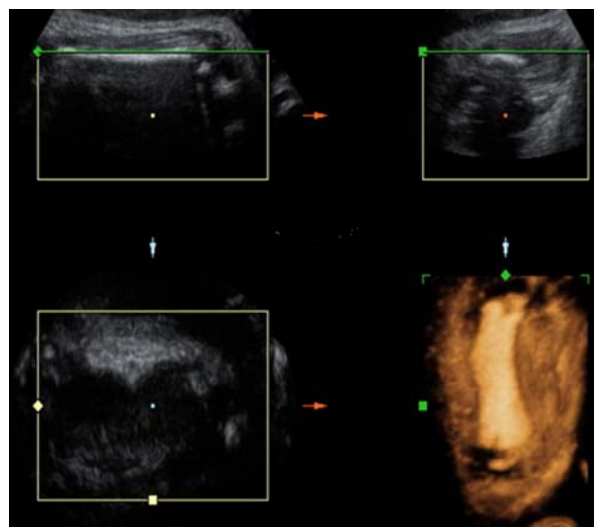

(b)

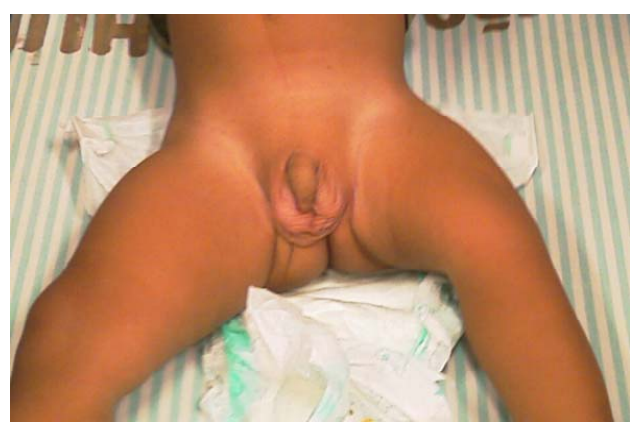

(c)

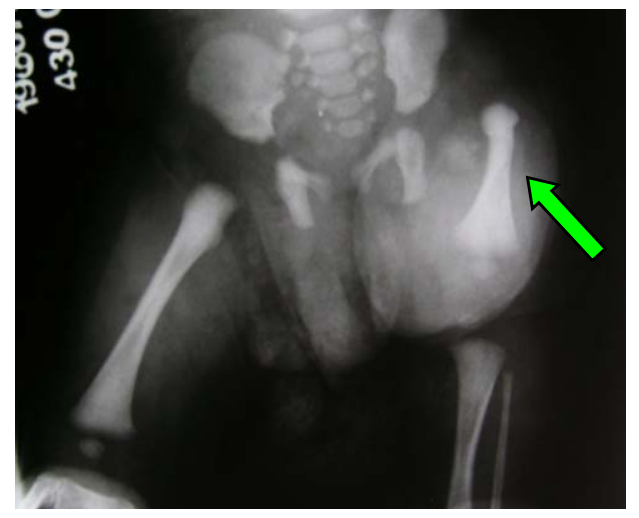

(d)

Figure 1. Case 03. (a) Prenatal Ultrasound (left short femur blue arrow); (b) tridimensional image (left short femur); (c) Post-natal view and (d) Post-natal radiography (left short femur green arrow).

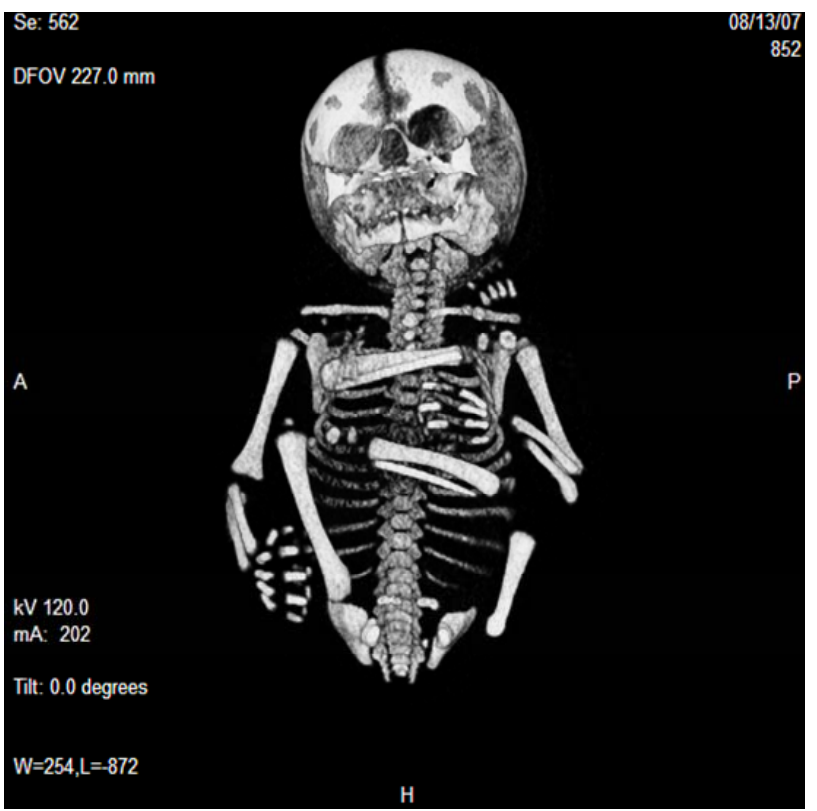

Figure 2. Computer tomography (short femur).

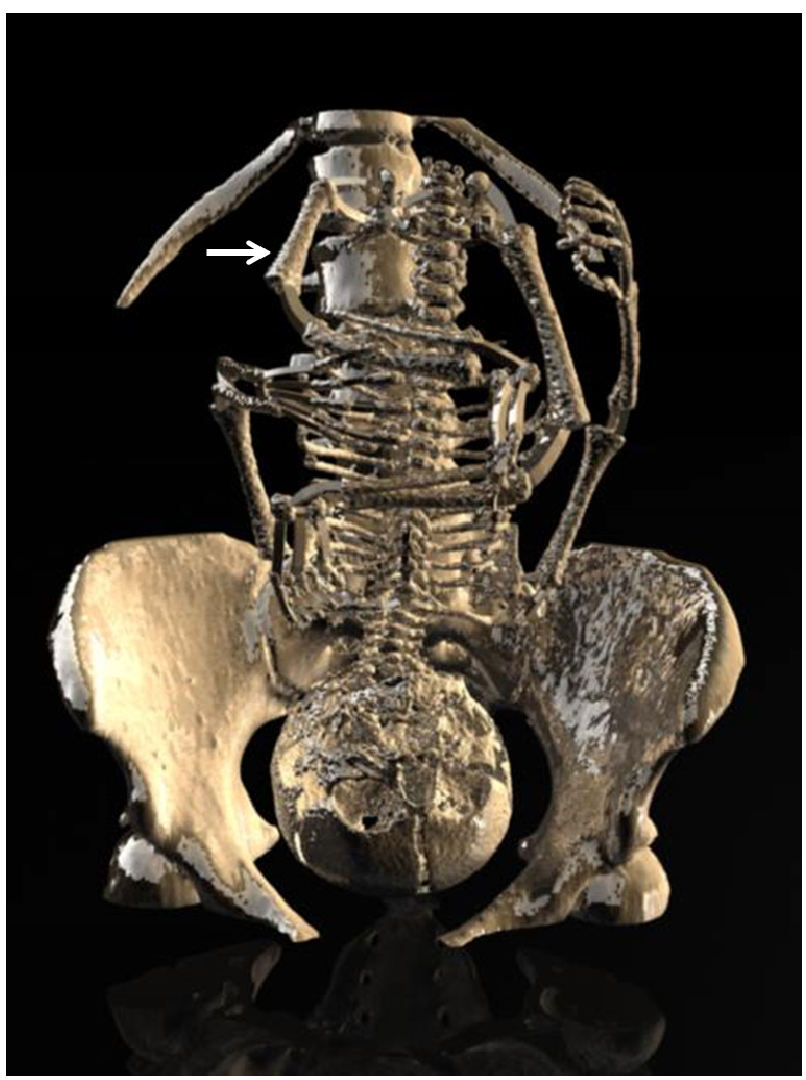

Figure 3. Tridimensional reconstruction by CT of the fetal skeleton demonstrating the short femur.

birth of the first, second, third and fourth newborns were 3790 g, 2950 g, 3570 g and 2970 g respectively. Physical examination and postnatal radiography confirmed the 2D-US and CT images. Congenital isolated short femur 
was also the diagnosis for these newborns. The first and second infants were both 2 years old and showed a 5.2and $6.0-\mathrm{cm}$ difference between limb lengths, respectively. The third infant was 1 year old and showed a $6.7-\mathrm{cm}$ difference between limb lengths. No other anomalies were found for all except for the fourth. He was born one week before the manuscript was written and he presented agenesis of the second finger of the shortened femur. All the children were waiting for prosthesis when the manuscript was submitted.

Our work complies with all regulations laid down by our country and we have obtained the necessary informed consent for all cases.

\section{Discussion}

PFFD is a congenital anomaly of the pelvis and proximal femur, characterized by hip deformity, several degrees of reduction and altered function of the involved lower extremity. PFFD is considered a sporadic condition, but a familial form has also been described [6]. The unilateral form is more common, occurring in 85 to $90 \%$ of the cases [7]. It has been suggested that PFFD is part of a development field defect including femur-fibula-ulna syndrome, fibula aplasia-hypoplasia and femoral hypoplasia/unusual facies syndrome [6]. Poor diabetic control, exposure to drugs (thalidomide), viral infection, radiation, focal ischemia and trauma between $4^{\text {th }}$ and $8^{\text {th }}$ week of gestation have been implicated as possible etiological factors in others cases. This disorder was classified into four groups by Aitken [8] (Table 1). This classification takes into account the anatomic relationship between the acetabulum and the proximal end of the femur, and has prognostic significance. PFFD is not associated with obstetric complications.

The management of proximal femoral focal deficiency requires a multidisciplinary team, which includes the pediatric orthopedic surgeon, prosthetists, and physical therapists. The goals of treatment of PFFD are to compensate for the functional deficits. Treatment must be indi- vidualized based on: leg length discrepancy, presence of foot deformities, adequacy of musculature, proximal joint stability. Options of surgical theraty are [19]:

1) Limb lengthening with or without contralateral epiphysiodesis-Indications: predicated limb length discrepancy of $<20 \mathrm{~cm}$ at maturit, stable hip and functional foot, femoral length $>50 \%$ of opposite side, femoral head present (Aiken classifications A \& B)

2) Femoral-pelvic fusion (Brow's procedure) - Indications: femoral head absent (Aiken classifications C \& D).

3) Van Ness rotationplastty-Indications: ipsilateral foot at level of contralateral knee, ankle with $>60 \%$ of motion, absent femoral head (Aiken classifications C \& D)

4) Amputation-Indications: femoral length $<50 \%$ of opposite side. Fit for prosthesis in 6 months to 1 year. Prenatal diagnosis is suspected by US screening when a discrepancy between the measurements of both femurs is found.

The challenge of antenatal diagnosis of skeletal dysplasia is generally presented in one of two ways: the incidental finding of a shortened, bowed, or anomalous extremity during a routine sonographic examination; or a patient who has delivered an infant with skeletal dysplasia and desires antenatal assessment in a subsequent pregnancy. The role of diagnostic imaging in prenatal investigation of skeletal dysplasia is to improve the differential diagnosis, in order to both predict lethality and identify anomalies early enough in pregnancy so that the diagnostic workup can be completed before the limit of fetal viability [6].

Routine conventional US is the main prenatal screening method for skeletal disorders, able to identify abnormalities in fetal bones, mainly shortening of the long bones [9,10]. Welder et al. [11] screened 12,453 patients in the second and third trimesters of pregnancy, and estimated the prevalence of skeletal dysplasia as detected by prenatal ultrasound as being 7.5 per 10,000 live births. However, only part of cases were accurately diagnosed

Table 1. Proximal femoral focal deficiency (PFFD) classification [8].

\begin{tabular}{l} 
Aitken class \\
\hline A shortened femur is present proximally, ending at or slightly above the level of the acetabulum. The femoral head is often \\
absent but later ossifies; femoral head presence is indicated by a well-developed acetabulum. Additionally, there is a sub- \\
trochanteric defect, which eventually ossifies and thereby establishes bony continuity. After ossification, there is usually a \\
residual subtrochanteric various deformity. \\
There is a more severe defect or absence of the proximal femur. This defect does not heal spontaneously. At skeletal ma- \\
turity, there is no connection between the femoral head and proximal femur; the end of the proximal femur is above the \\
acetabulum. The femoral head, although present, may have delayed ossification, and there is often a bony tuft on the proxi- \\
mal end of the shaft. \\
Absent femoral head that does not ossify and a markedly dysplastic acetabulum. The class C femoral shaft is shorter than in \\
a person with class B, in whom the entire proximal femur, including the trochanters, does not develop. \\
The most severe form, there is a severely shortened shaft, which often has only an irregularly ossified tuft of bone proximal \\
to the distal femoral epiphysis. No acetabulum is present because the lateral pelvic wall is flat.
\end{tabular}


by conventional two-dimensional ultrasound (2D-US) [12-17] (Table 2).

According to Ruano et al., 3D-US and CT both detect fetal bone abnormalities more accurately than 2D-US does [18]. When rendering algorithms for reconstruction of the fetal skeleton are available, observation of phenotypic features that are not currently detectable by 2D-US will be possible.

The lower cost and absence of fetal exposition to radiation are the great advantages of 3D-US. If the fetus is examined early enough during pregnancy, image of the entire skeleton can be included within the region of interest and a panoramic visualization can be obtained. However, diagnosis may still be missed, since the phenotypic characteristics of some skeletal dysplasia are not observable unless in later pregnancy. Furthermore, 3DUS is more dependent on amniotic fluid volume and fetal position.

CT has been proposed [17] as an adjunctive imaging modality for prenatal diagnosis of skeletal dysplasia. This technique provides attenuation-based X-ray images, without superposition on those of the maternal skeleton, which are very similar to radiological images obtained postnatally. Ruano et al. [18] compared the phenotypic characteristics of tree skeletal dysplasias (achondroplasia, osteogenesis imperfecta, and chondrodysplasia punctata) as visualized by prenatal CT, 3D-US, and 2D-US. According to those authors CT identified more postnatal skeletal findings than 3D-US did.

\section{Conclusion}

In conclusion, CT images can reveal the entire fetal skeleton, whereas the 3D-US ones show only specific parts of the fetus, especially in the third trimester of pregnancy. We speculate that the PFFD classification is more feasible by CT than 2D-US or 3D-US, and it could help the treatment strategies and prognosis after birth.

\section{Acknowledgements}

The authors acknowledge Dr Paulo Boschcov, former

Table 2. Accuracy of prenatal ultrasound for diagnosis of skeletal dysplasias.

\begin{tabular}{ccccc}
\hline Author & Year & Number of Cases & \multicolumn{2}{c}{ Correct Diagnosis } \\
\hline Gordienko [13] & 1996 & 26 & 9 & $34 \%$ \\
Gaffney [14] & 1998 & 35 & 11 & $31 \%$ \\
Tretter [15] & 1998 & 27 & 13 & $48 \%$ \\
Hersh [16] & 1998 & 23 & 11 & $48 \%$ \\
Dorey [17] & 2000 & 47 & 28 & $60 \%$ \\
Parilla [12] & 2003 & 31 & 20 & $65 \%$ \\
\hline
\end{tabular}

professor at UNIFESP, whose suggestions contributed to improve the quality of the final version of the manuscript.

\section{REFERENCES}

[1] D. E. Westberry and J. R. Davids, "Proximal Focal Femoral Deficiency (PFFD): Management Options and Controversies," Hip International, Vol. 19, Suppl. 6, 2009, pp. S18-25.

[2] H. Werner, J. R. Lopes dos Santos, R. Fontes, E. L. Gasparetto, P. A. Daltro, Y. Kuroki and R. C. Domingues, "The Use of Rapid Prototyping Didactic Models in the Study of Fetal Malformations," Ultrasound in Obstetrics \& Gynecology, Vol. 32, No. 7, 2008, pp. 955-958. doi:10.1002/uog.6253

[3] M. Cassart, A. Massez, T. Cos, L. Tecco, D. Thomas, N. Van Regemorter and F. Avni, "Contribution of ThreeDimensional Computed Tomography in the Assessment of Fetal Skeletaldysplasia," Ultrasound in Obstetrics \& Gynecology, Vol. 29, No. 3, 2007, pp. 537-543. doi:10.1002/uog.4001

[4] C. Hamanashi, "Congenital Short Femur. Clinical, Genetic and Epidemiological Comparison of the Naturally Occurring Condition with That Caused by Thalidomide," The Journal of Bone \& Joint Surgery, Vol. 62, No. 3, 1980, pp. 307-320.

[5] J. Diaz-Faes and A. I. Cereijo, "Femur Corto Congenito: Frecuencia en el ECEMC, Etiologia y Tratamiento," Revista Dismorfol Epidemiology, Vol. 3, 1992, pp. 19-28.

[6] L. F. Gonçalves, J. P. Kusanovic, F. Gotsch, J. Espinoza and R. Romero, "The Fetal Musculoskeletal System," In: W. P. Callen, Ed., Ultrasonography in Obstetrics and Gynecology, 5th Edition, Saunders, New York, 2008, pp 419-492.

[7] P. Jeanty and G. Kleinman, "Proximal Femoral Focal Deficiency,” Journal of Ultrasound in Medicine, Vol. 8, 1989, pp. 639-642.

[8] G. T. Aitken, "Proximal Femoral Focal Deficiency-Definition, Classification and Management,” Proximal Femoral Focal Deficiency: A Congenital Anomaly, National Academy of Sciences, Washington DC, 13 June 1968, pp. $1-22$.

[9] M. P. De Pellegrin, W. G. Mackenzie and H. T. Harcke, "Ultrasonographic Evaluation of Hip Morphology in Osteochondrodysplasias," Journal of Pediatric Orthopaedics, Vol. 20, 2000, pp. 588-593. doi:10.1097/01241398-200009000-00009

[10] D. Krakow, R. S. Lachman and D. L. Rimoin, "Guidelines for the Prenatal Diagnosis of Fetal Skeletal Dysplasias," Genetics in Medicine, Vol. 11, No. 2, 2009, pp. 127-133.

[11] B. M. Weldner, P. H. Persson and S. A. Ivarsson, "Prenatal Diagnosis of Dwarfism by Ultrasound Screening," Archives of Disease in Childhood, Vol. 60, No. 11, 1985, pp. 1070-1072. doi:10.1136/adc.60.11.1070

[12] B. V. Parilla, E. A. Leeth, M. P. Kambich, P. Chilis and S. N. MacGregor, “Antenatal Detection of Skeletal Dyspla- 
sias,” Journal of Ultrasound in Medicine, Vol. 22, No. 3, 2003, pp. 255-258.

[13] I. Y. Gordienko, E. Y. Grechanina, N. I. Sopko, E. N. Tarapurova and L. P. Mikchailets, "Prenatal Diagnosis of Osteochondrodysplasias in High Risk Pregnancy," American Journal of Medical Genetics, Vol. 63, No. 1, 1996, pp. 90-97. doi:10.1002/(SICI)1096-8628(19960503)63:1<90::AIDAJMG18>3.0.CO;2-P

[14] G. Gaffney, N. Manning, P. A. Boyd, V. Rai, S. Gould and P. Chamberlain, "Prenatal Sonografic Diagnosis of Skeletal Dysplasias-A Report of the Diagnosis and Prognostic Accuracy in 35 Cases,” Prenatal Diagnosis, Vol. 18, No. 4, 1998, pp. 357-362. doi:10.1002/(SICI)1097-0223(199804)18:4<357::AID-P D276>3.0.CO;2-0

[15] A. E. Tretter, R. C. Saunders, C. M. Meyers, J. S. Dungan, K. Grumbach, C. C. Sun, A. B. Campbell and E. A. Wulfsberg, "Antenatal Diagnosis of Lethal Skeletal Dysplasias,” American Journal of Medical Genetics, Vol. 75, No. 5, 1998, pp. 518-522.
doi:10.1002/(SICI)1096-8628(19980217)75:5<518::AIDAJMG12>3.0.CO;2-N

[16] J. H. Hersh, B. Angle, M. Pietrantoni, V. D. Cook, J. A. Spinnato, A. L. Clark, J. T. Kurtzman, R. W. Bendon and A. Gerassimides, "Predictive Value of Fetal Ultrasonography in the Diagnosis of a Lethal Skeletal Dysplasia," Southern Medical Journal, Vol. 91, No. 12, 1998, pp. 1137-1142. doi:10.1097/00007611-199812000-00008

[17] B. Doray, R. Favre, B. Viville, B. Langer, M. Dreyfus and C. Stoll, "Prenatal Sonographic Diagnosis of Skeletal Dysplasias. A Report of 47 Cases,” Annales de Génétique, Vol. 43, No. 3-4, 2000, pp. 163-169. doi:10.1016/S0003-3995(00)01026-1

[18] R. Ruano, M. Molho, J. Roume and Y. Ville, "Prenatal Diagnosis of Skeletal Dysplasias by Combining TwoDimensional and Three-Dimensional Ultrasound and Three-Dimensional Helical Computer Tomography," Ultrasound in Obstetrics \& Gynecology, Vol. 24, 2004, pp. 134-140. doi:10.1002/uog.1113 